TRANSACTIONS OF THE

AMERICAN MATHEMATICAL SOCIETY

Volume 329, Number 1, January 1992

\title{
A DECOMPOSITION THEOREM FOR THE SPECTRAL SEQUENCE OF LIE FOLIATIONS
}

\author{
JESÚS A. ALVAREZ LÓPEZ
}

\begin{abstract}
For a Lie $\mathfrak{g}$-foliation $\mathscr{F}$ on a closed manifold $M$, there is an "infinitesimal action of $\mathfrak{g}$ on $M$ up to homotopy along the leaves", in general it is not an action but defines an action of the corresponding connected simply connected Lie group $\mathfrak{S}$ on the term $E_{1}$ of the spectral sequence associated to $\mathscr{F}$. Even though $E_{1}$ in general is infinite-dimensional and non-Hausdorff (with the topology induced by the $\mathscr{C}^{\infty}$-topology), it is proved that this action can be averaged when $\mathfrak{S}$ is compact, obtaining a tensor decomposition theorem of $E_{2}$. It implies duality in the whole term $E_{2}$ for Riemannian foliations on closed oriented manifolds with compact semisimple structural Lie algebra.
\end{abstract}

\section{INTRODUCTION}

The spectral sequence $\left(E_{i}, d_{i}\right)$ associated to a smooth foliation $\mathscr{F}$ on a manifold $M$ is defined for example in [Sa, KT2, and Se2]. The $\mathscr{C}^{\infty}$-topology in the space of differential forms induces in $E_{1}$ a topology which in general is not Hausdorff [Ha1], obtaining two new bigraded differential algebras: The closure $\overline{O_{E_{1}}}$ of the trivial subspace in $E_{1}$ and the quotient $\mathscr{E}_{1}=E_{1} / \overline{O_{E_{1}}}[\mathrm{He}$ and A2].

If $\mathscr{F}$ is Riemannian and $M$ is compact there are several papers studying the finite-dimensional character and the duality in $E_{2}, \mathscr{E}_{2}=H\left(\mathscr{E}_{1}\right)$ and $H\left(\overline{O_{E_{1}}}\right)$, or in parts of them: [Sa, EH, ESH, Se1, Se2, He, KT1-KT3, A1 and A2]. For this type of foliations, with $M$ oriented, it is proved in [A2] that the de Rham duality map induces in $H\left(\overline{O_{E_{1}}}\right)$ and $\mathscr{E}_{2}$ different types of duality, so the possibility of obtaining duality in $E_{2}$ depends on the properties of the canonical long exact sequence which relates these three homologies. For example, in [A2] it is proved that $E_{2} \cong \mathscr{E}_{2}$ when the leaves are compact.

In the present paper this last result is generalized (§4), obtaining that $E_{2} \cong \mathscr{E}_{2}$ when the structural Lie algebra of $\mathscr{F}$ is compact and semisimple. Using the structure theorems of P. Molino for Riemannian foliations [Mo] its proof is reduced to the case of Lie foliations, where it is a consequence of the main result of this paper ( $(3)$ : For Lie $\mathfrak{g}$-foliations on manifolds with $\mathfrak{g}$ compact and semisimple we have $E_{2} \cong E_{2}^{0, \cdot} \otimes H^{\cdot}(\mathfrak{g})$. The analogous decomposition for $\mathscr{E}_{2}$ is proved in [A2] with easier arguments.

Let $\mathscr{F}$ be a Lie $\mathfrak{g}$-foliation on a compact manifold $M$, and let $\mathfrak{S}$ be the connected simply connected Lie group with Lie algebra $\mathfrak{g}$. By choosing a com-

Received by the editors October 31, 1988.

1980 Mathematics Subject Classification (1985 Revision). Primary 57R30.

(C) 1992 American Mathematical Society $0002-9947 / 92 \$ 1.00+\$ .25$ per page 
plement $\nu \subset T M$ of the subbundle of vectors tangent to $\mathscr{F}$ we can define a smooth map $\phi: M \times \mathfrak{g} \rightarrow M(\S 1)$, which induces an infinitesimal action only if $\nu$ is completely integrable. So $\phi$ does not induce an action of $\mathfrak{S}$ neither on $M$ nor on its de Rham complex $A(M)$, but it induces an action on $E_{1}$. The proof of the above tensor decomposition of $E_{1}$ follows directly whenever the above action can be "averaged". If $\mathfrak{g}$ is compact and semisimple then $\mathfrak{S}$ is compact, but even in this case an averaging process cannot be easily made because, in general, $E_{1}$ is an infinite-dimensional non-Hausdorff topological vector space, (the integration of continuous functions of $\mathfrak{S}$ to $E_{1}$ is not well defined).

The dual action of $\mathfrak{S}$ on $E_{1}^{\prime}$ can be averaged [B], but in this case we obtain the tensor decomposition of $H\left(E_{1}^{\prime}\right) \equiv \mathscr{E}_{2}^{\prime} \cong \mathscr{E}_{2}$. Thus it would be another proof of Theorem (9.10) of [A2].

For the "basic complex", $E_{1}^{\cdot, 0}$, and the "transverse complex", $E_{1}^{\cdot, p} \quad(p=$ $\operatorname{dim}(\mathscr{F}))$, the situation is much simpler, for they can be identified with a subspace of $A(\mathfrak{S})$ and a quotient of $A_{c}(\mathfrak{S})$ respectively, where the above action of $\mathfrak{S}$ is given by the left translations [ $\mathrm{Ha} 1$ and $\mathrm{He}$ ]. So, the averaging process on $E_{1}^{\cdot, 0}$ and $E_{1}^{\cdot, p}$ can be easily made when $\mathfrak{g}$ is compact or nilpotent, obtaining $E_{2}^{\cdot, 0} \cong E_{2}^{\cdot, p} \cong H^{\cdot}(\mathfrak{g})$.

The main part of this paper is devoted to obtain an averaging process of the above action on the whole $E_{1}$ when $\mathfrak{g}$ is compact and semisimple. To do that, some parts of measure zero are modified in the closure of the domain of injectivity of the exponential map to define new compact spaces $(\S 2)$, so that $\phi$ can be "lift" to them, where the failure of being "like an action" can be controlled $(\S 3)$. Then, by integrating maps induced by the lifting of $\phi$ on those compact spaces, we obtain an operator on $A(M)$ which defines an "averaging retraction" of $E_{1}$ onto its subspace of elements that are invariant by the action of $\mathfrak{S}$. This retraction induces an isomorphism in cohomology from which the above decomposition theorem follows.

From the arguments of this proof, when $\mathscr{F}$ is orientable, we obtain an explicit expression of the volume form along the leaves induced by an orientation and the restriction to the leaves of a Riemannian metric on $M$ for which all the leaves of $\mathscr{F}$ are minimal submanifolds.

I wish to express my deep gratitude to Professor X. Masa and Professor E. Macias for useful comments.

\section{Preliminaries}

Let $M$ be a smooth manifold which carries a smooth foliation $\mathscr{F}$ of dimension $p$ and codimension $q$, and let $T \mathscr{F} \subset T M$ be the subbundle of vectors of $M$ tangent to $\mathscr{F}$.

The de Rham differential algebra $(A(M), d)$ (or simply $(A, d)$ ) of $M$ is filtered by differential ideals so that a differential form of degree $r$ is said to be of filtration $\geq k$ if it vanishes whenever $r-k+1$ of the vectors are tangent to $\mathscr{F}$. In this way we obtain the spectral sequence $\left(E_{i}(\mathscr{F}), d_{i}\right)$ (or simply $\left.\left(E_{i}, d_{i}\right)\right)$ which converges after a finite number of steps to the de Rham cohomology of $M$.

For each subbundle $\nu \subset T M$, complementary of $T \mathscr{F}$, we bigrade $A$ by setting

$$
A^{u, v}=\Gamma\left(\Lambda^{v} T^{*} \mathscr{F} \otimes \Lambda^{u} \nu^{*}\right)
$$


for $u, v$ integers, obtaining the decomposition of $d$ as sum of the bihomogeneous operators $d_{0,1}, d_{1,0}$ and $d_{2,-1}$, where the double subindices denote the corresponding bidegrees, verifying

$$
\begin{gathered}
d_{0,1}^{2}=d_{2,-1}^{2}=d_{0,1} \circ d_{1,0}+d_{1,0} \circ d_{0,1}=0, \\
d_{1,0} \circ d_{2,-1}+d_{2,-1} \circ d_{1,0}=d_{1,0}^{2}+d_{2,-1} \circ d_{0,1}+d_{0,1} \circ d_{2,-1}=0 .
\end{gathered}
$$

And we have the following canonical identities of bigraded differential algebras

$$
\left(E_{0}, d_{0}\right) \equiv\left(A, d_{0,1}\right), \quad\left(E_{1}, d_{1}\right) \equiv\left(H\left(A, d_{0,1}\right), d_{1,0 *}\right) .
$$

$(A, d)$ is a topological differential algebra with the $\mathscr{C}^{\infty}$-topology, then each $\left(E_{i}, d_{i}\right)$ is another topological differential algebra with the induced topology and the identities (1.2) are also topological.

$E_{1}$ in general is not Hausdorff [Hal] obtaining the bigraded differential algebras $\overline{O_{E_{1}}}$ (the closure in $E_{1}$ of the trivial subspace) and $\mathscr{E}_{1}=E_{1} / \overline{O_{E_{1}}}$. Let $\mathscr{E}_{2}=H\left(\mathscr{E}_{1}\right)$.

If $\mathscr{F}$ is Riemannian and $M$ compact then $E_{2}, \mathscr{E}_{2}$, and $H\left(\overline{O_{E_{1}}}\right)$ are of finite dimension [Se2, A1 and A2], and if $M$ is also oriented we have the duality isomorphisms [A2]

$$
\mathscr{E}_{2}^{u, v} \cong \mathscr{E}_{2}^{q-u, q-v}, \quad H^{u, v}\left(\overline{O_{E_{1}}}\right) \cong H^{q-u-1, p-v+1}\left(\overline{O_{E_{1}}}\right)
$$

for $u, v$ integers, obtaining the canonical isomorphism

$$
E_{2}^{q, \cdot} \cong \mathscr{E}_{2}^{q, \cdot}
$$

Suppose that $\mathscr{F}$ is a Lie $\mathfrak{g}$-foliation and $M$ is compact. If $\mathfrak{S}$ is the simply connected Lie group with Lie algebra $\mathfrak{g}$ then [Mo] there exists a covering map $\pi: \widetilde{M} \rightarrow M$ and a fibre bundle $D: \widetilde{M} \rightarrow \mathfrak{S}$ such that:

(i) The leaves of $\pi^{*} \mathscr{F}$ are the fibres of $D$.

(ii) There exists an injective homomorphism $h: \operatorname{Aut}(\pi) \rightarrow \mathfrak{S}$ so that $D$ is $h$-equivariant $(D \circ \zeta(\tilde{x})=h(\zeta) \cdot D(\tilde{x})$ for $\tilde{x} \in \widetilde{M}$ and $\zeta \in \operatorname{Aut}(\pi))$.

Choose $\nu \subset T M$ as above and the corresponding bigradation in $A$. Let $\tilde{\nu}=\pi^{*} \nu \subset T \widetilde{M}$ and $\widetilde{\mathscr{F}}=\pi^{*} \mathscr{F}$. For each field $X \in \mathfrak{g}$ there exists a unique field $\tilde{X}^{\nu} \in \Gamma \tilde{\nu}$ such that $D_{*} \circ \tilde{X}^{\nu}=X \circ D$. Since $D$ is $h$-equivariant $\widetilde{X}^{\nu}$ is $\operatorname{Aut}(\pi)$-invariant, so it determines a field $X^{\nu} \in \Gamma \nu \subset \mathfrak{X}(M)$ which is an infinitesimal transformation of $\mathscr{F}$. Let $\theta_{X}$ and $i_{X}$ be the Lie derivative $\mathscr{L}_{X^{\nu}}$ and the interior product $i_{X^{\nu}}$ respectively, obtaining

$$
\begin{gathered}
d_{0,1} \circ i_{X}+i_{X} \circ d_{0,1}=0, \quad\left(\theta_{X}\right)_{0,0} \circ d_{0,1}=d_{0,1} \circ\left(\theta_{X}\right)_{0,0}, \\
i_{[X, Y]}=\left(\theta_{X}\right)_{0,0} \circ i_{Y}-i_{Y} \circ\left(\theta_{X}\right)_{0,0}, \quad\left(\theta_{X}\right)_{0,0}=d_{1,0} \circ i_{X}+i_{X} \circ d_{1,0}, \\
\left(\theta_{[X, Y]}\right)_{0,0}=\left(\theta_{X} \circ \theta_{Y}-\theta_{Y} \circ \theta_{X}\right)_{0,0}-d_{0,1} \circ i_{\Omega(X \Lambda Y)}-i_{\Omega(X \Lambda Y)} \circ d_{0,1},
\end{gathered}
$$

where $\Omega: \Lambda^{2} \mathfrak{g} \rightarrow \Gamma T \mathscr{F}$ is the linear map defined by $\Omega(X \Lambda Y)=\left[X^{\nu}, Y^{\nu}\right]-$ $[X, Y]^{\nu}$. Therefore we get the operation $\left(\mathfrak{g}, i_{1}, \theta_{1}, E_{1}, d_{1}\right)$, where $i_{1_{X}} \equiv i_{X^{*}}$ and $\theta_{1_{X}} \equiv\left(\theta_{X}\right)_{0,0 *}$ by (1.2), with the algebraic connection $\chi: \mathfrak{g}^{*} \rightarrow E_{1}^{1,0} \subset$ $A^{1,0}$ where $\chi(\alpha)$ is determined by $\pi^{*} \chi(\alpha)=D^{*} \alpha$ for each $\alpha \in \mathfrak{g}^{*} \subset A^{1}(\mathfrak{S})$. Then it follows that [Ma and A2]

$$
E_{2}^{u, v} \cong H^{u}\left(\mathfrak{g} ; \theta_{1}: \mathfrak{g} \rightarrow \operatorname{End}\left(E_{1}^{0, v}\right)\right) \quad \text { for } u, v \text { integers } .
$$


The above operation and algebraic connection induces an operation of $\mathfrak{g}$ on $\mathscr{E}_{1}$ with the corresponding algebraic connection and analogous consequences.

For each (complete) vector field $X$ we denote by $X_{t}(t \in \mathbb{R})$ the corresponding one-parameter group of transformations. Then we have the smooth maps $\phi: M \times \mathfrak{g} \rightarrow M$ and $\tilde{\phi}: \widetilde{M} \times \mathfrak{g} \rightarrow \widetilde{M}$, depending on $\tilde{\nu}$, defined by $\phi(x, X)=X_{1}^{\nu}(x)$ and $\tilde{\phi}(\tilde{x}, X)=\widetilde{X}_{1}^{\nu}(\tilde{x})$, obtaining the commutativity of the diagrams

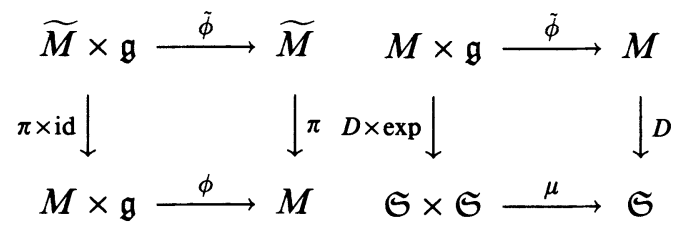

where $\mu$ is the operation of $\mathfrak{S}$. Let $\phi_{X}=\phi(\cdot, X): M \rightarrow M$ for each $X \in \mathfrak{g}$.

\section{COMPACT SPACES ASSOCIATED TO A COMPACT LIE GROUP}

Let $N$ be a connected complete Riemannian manifold. For each $x \in N$ let $C(x) \subset N$ and $C^{*}(x) \subset T_{x} N$ be the corresponding cut locus and tangential cut locus respectively [Ko and $\mathrm{Kl}$ ], let $B^{*}(x)$ be the radial domain in $T_{x} N$ bounded by $C^{*}(x)$ and let $B(x)=\exp _{x}\left(B^{*}(x)\right)$.

Proposition 2.1 [Ko and Kl]. (i) $C(x)=\partial B(x)=N-B(x)$.

(ii) $\exp _{x}: B^{*}(x) \rightarrow B(x)$ is a diffeomorphism.

(iii) $C(x)$ and $C^{*}(x)$ have Lebesgue measure zero.

(iv) If $N$ is compact then so is $\overline{B^{*}(x)}$.

Let $\mathfrak{S}$ be a connected compact Lie group with Lie algebra $\mathfrak{g}$ and consider the canonical biinvariant metric on $\mathfrak{S}$ (Chapter 6 of [P]). For the identity element $e \in \mathfrak{S}$ let $B^{*}=B^{*}(e) \subset \mathfrak{g}, B=B(e) \subset \mathfrak{S}$ and $\log =\exp ^{-1}: B \rightarrow B^{*}$. Then we have the compact space

$$
F=\left\{(X, Y, Z) \in \overline{B^{* 3}} / \exp (X) \cdot \exp (Y)=\exp (Z)\right\} \subset \mathfrak{g}^{3},
$$

and for each $X \in \overline{B^{*}}$ we also have the compact space

$$
F_{X}=\left\{(Y, Z) \in \mathfrak{g}^{2} /(X, Y, Z) \in F\right\} \subset \mathfrak{g}^{2} .
$$

We can speak of smoothness of geometrical objects on $F$ (resp. $F_{X}$ ) as subspace of $\mathfrak{g}^{3}$ (resp. $\left.\mathfrak{g}^{2}\right)$.

Let $i: \mathfrak{g}^{2} \rightarrow \mathfrak{g}^{2}$ be the involution $(Y, Z) \mapsto(Z, Y)$ and let $\mathrm{pr}_{1}: \mathfrak{g}^{2} \rightarrow \mathfrak{g}$ be the canonical first projection. Denoting $a=\exp (X)$ we have the smooth map

$$
\begin{aligned}
& \mathscr{J}_{X}: B \cap L_{a}^{-1} B \rightarrow F_{X} \\
& g \mapsto(\log (g), \log (a \cdot g))
\end{aligned}
$$

and let $W_{X}$ be its image.

Proposition 2.2. (i) $W_{X}$ is open in $F_{X}$ and $\mathscr{I}_{X}: B \cap L_{a}^{-1} B \rightarrow W_{X}$ is a diffeomorphism.

(ii) In general $\overline{W_{X}} \neq F_{X} \cdot \overline{W_{X}}$ is formed by the pairs $(Y, Z) \in F_{X}$ such that

$$
Z \in \overline{B^{*} \cap \exp ^{-1} L_{a}^{-1} \exp \left(U \cap B^{*}\right)}
$$

for all neighborhoods $U$ of $Y$ in $\mathfrak{g}$. 
(iii) $i\left(F_{X}\right)=F_{-X}$ and we have the commutative diagram

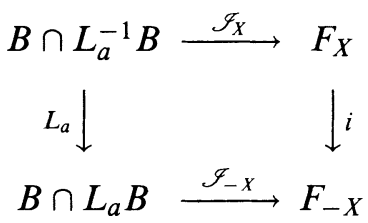

Proof. (i) We have $W_{X}=F_{X} \cap\left(\log \left(B \cap L_{a}^{-1} B\right) \times \mathfrak{g}\right)$ which is open in $F_{X}$, and it is easy to check that $\exp \circ \mathrm{pr}_{1}: W_{X} \rightarrow B \cap L_{a}^{-1} B$ is the inverse map of $\mathscr{I}_{X}: B \cap L_{a}^{-1} B \rightarrow W_{X}$.

(ii) If $Y$ and $Z$ are different points in $\overline{B^{*}}$ such that $\exp (Y)=\exp (Z)$ then $(Y, Z) \in F_{0}-\overline{W_{0}}$.

Let $(Y, Z) \in \overline{W_{X}}$ and let $U$ be a neighborhood of $Z$ in $\mathfrak{g}$. Take a sequence $\left(\left(Y_{n}, Z_{n}\right)\right)_{n \in \mathbf{N}}$ in $W_{X}$ converging to $(Y, Z)$. We can assume that $Y_{n} \in U$ for all $n \in \mathbb{N}$ obtaining that $Z_{n} \in B^{*} \cap \exp ^{-1} L_{a} \exp \left(U \cap B^{*}\right)$, so (2.1) is verified .

Let $(Y, Z) \in F_{X}$ such that $(2.1)$ is verified for all neighborhood $U$ of $Y$. If $\left\{U_{n}\right\}_{n \in \mathbb{N}}$ and $\left\{V_{n}\right\}_{n \in \mathbb{N}}$ are neighborhood bases of $Y$ and $Z$ respectively then for each $n \in \mathbb{N}$ there exists a point $Z_{n} \in V_{n} \cap B^{*} \cap \exp ^{-1} L_{a} \exp \left(U_{n} \cap B^{*}\right)$, obtaining that $g_{n}=a^{-1} \cdot \exp \left(Z_{n}\right) \in B \cap L_{a}^{-1} B$ and $\left(\mathscr{I}_{X}\left(g_{n}\right)\right)_{n \in \mathbb{N}}$ converges to $(Y, Z)$, so $(Y, Z) \in \overline{W_{X}}$.

(iii) Since $\exp (-X)=a^{-1}$ we have $i\left(F_{X}\right)=F_{-X}$, and the commutativity of (2.2) has a straightforward verification.

For $X, Y \in \overline{B^{*}}$ we have the open subset $W_{X, Y}=\mathscr{J}_{X}\left(B \cap L_{a}^{-1} B \cap L_{b}^{-1} B\right) \subset$ $F_{X}$, where $a=\exp (X)$ and $b=\exp (Y)$, obtaining the diffeomorphism $\mathscr{I}_{X, Y}=$ $\mathscr{I}_{Y} \circ \mathscr{J}_{X}^{-1}: W_{X, Y} \rightarrow W_{Y, X}$

Assume $\mathfrak{S}$ oriented and let $\Delta$ be the unique biinvariant volume form on $\mathfrak{S}$ such that $\int_{\mathfrak{S}} \Delta=1$, which induces a Haar measure $\mu$ on $\mathfrak{S}$. Then, for each $X \in \overline{B^{*}}$ let $\mu_{X}$ be the Borel measure on $F_{X}$, concentrated on $W_{X}$, where it corresponds to $\mu$ by $\mathscr{I}_{X}$.

Proposition 2.3. We have:

(i) $\mu_{X}\left(F_{X}\right)=\mu_{X}\left(W_{X}\right)=\mu_{X}\left(W_{X, Y}\right)=\mu\left(B \cap L_{a}^{-1} B \cap L_{b}^{-1} B\right)=\mu\left(B \cap L_{a}^{-1} B\right)=$ $\mu(\mathfrak{S})=1$.

(ii) $\mu_{X}$ corresponds to $\mu_{-X}$ by $i: F_{X} \rightarrow F_{-X}$.

(iii) $\mu_{X}$ corresponds to $\mu_{Y}$ by $\mathscr{J}_{X, Y}: W_{X, Y} \rightarrow W_{Y, X}$.

Proof. (i) follows from Proposition 2.1, (ii) follows from the commutativity of (2.2), and (iii) is true because $\mathscr{I}_{X, Y}=\mathscr{I}_{Y} \circ \mathscr{J}_{X}^{-1}$.

\section{Decomposition of $E_{2}$}

Let $\mathscr{F}$ be a Lie $\mathfrak{g}$-foliation with $\mathfrak{g}$ compact and semisimple, then the simply connected Lie group $\mathfrak{S}$ with Lie algebra $\mathfrak{g}$ is compact $(6.22$ of $[\mathrm{P}])$. Examples of such a $\mathfrak{g}$ are $\mathfrak{s u}(n)$ and $\mathfrak{s p}(n)$ for all $n \in \mathbb{N}$, and $\mathfrak{s o}(n)$ for $n>2$ (6.28, 6.47 and 6.48 of [P]). The notation of the $\S \S 1$ and 2 remains here. Then, for a fixed subbundle $\nu \subset T M$, complementary of $T \mathscr{F}$, we define the operators $\rho$ and $\lambda$ on $A$ by setting

$$
\rho(\alpha)=\int_{\overline{B^{*}}} \phi_{X}^{*} \alpha \cdot \Delta^{*}(X), \quad \lambda(\alpha)=\int_{\overline{B^{*}}} \Phi_{X} \alpha \cdot \Delta^{*}(X),
$$


where $\Delta^{*}=\exp ^{*} \Delta$ and $\Phi_{X}$ is the homogeneous operator of degree -1 on $A$ associated to the homotopy $\phi_{t X}(t \in I=[0,1]) . \quad \rho$ and $\lambda$ are linear homogeneous of degrees 0 and -1 respectively, being $\rho-\mathrm{id}=d \circ \lambda+\lambda \circ d$. Moreover, since $\phi_{t X}$ preserves the foliation (because $X^{\nu}$ is an infinitesimal transformation of $\mathscr{F}$ ) $\Phi_{X}$ reduces the filtration at most by a unity, so $\rho$ is filtration-preserving and $\lambda$ reduces the filtration at most by a unity. Therefore they induce bihomogeneous linear operators $\rho_{1}$ and $\lambda_{1}$ on $E_{1}$ of bidegrees $(0,0)$ and $(-1,0)$ respectively such that $\rho_{1}-\mathrm{id}=\lambda_{1} \circ d_{1}+d_{1} \circ \lambda_{1} \quad\left(\rho_{1} \equiv \rho_{0,0 *}\right.$ and $\lambda_{1} \equiv \lambda_{-1,0 *}$ by $\left.(1.2)\right)$.

If $F$ is the compact space associated to $\mathfrak{S}(\S 2)$ we define the continuous maps $\sigma, \eta: F \times I \rightarrow \mathfrak{S}$ by setting

$$
\eta(\xi, t)= \begin{cases}\exp (2 t X) & \text { if } t \in I_{1}=[0,1 / 2], \\ \exp (X) \cdot \exp ((2 t-1) Y) & \text { if } t \in I_{2}=[1 / 2,1],\end{cases}
$$

where $\xi=(X, Y, Z) \in F, \sigma$ is smooth and so are the restrictions of $\eta$ to each $F \times I_{i}(i=1,2)$.

For each $\xi_{0}=\left(X_{0}, Y_{0}, Z_{0}\right) \in F$ we have the paths $\sigma\left(\xi_{0}, \cdot\right), \eta\left(\xi_{0}, \cdot\right): I \rightarrow \mathfrak{S}$ of the identity element $e \in \mathfrak{S}$ to $\exp \left(Z_{0}\right)$, the first one is smooth and the second one has smooth restrictions to each $I_{i}$. So there exists a homotopy $H_{\xi_{0}}: I \times I \rightarrow$ $\mathfrak{S}$ of $\sigma\left(\xi_{0}, \cdot\right)$ to $\eta\left(\xi_{0}, \cdot\right)$, relative to $\{0,1\}$, with smooth restrictions to each $I_{i} \times I$.

We define the maps $\sigma_{\xi_{0}}, \eta_{\xi_{0}}: F \times I \rightarrow \mathfrak{S}$ by setting

$$
\sigma_{\xi_{0}}(\xi, t)=\sigma\left(\xi_{0}, t\right)^{-1} \cdot \sigma(\xi, t), \quad \eta_{\xi_{0}}(\xi, t)=\eta\left(\xi_{0}, t\right)^{-1} \cdot \eta(\xi, t),
$$

and let $U$ be a contractile neighborhood of $e$ in $\mathfrak{S}$. Thus, since $\sigma_{\xi_{0}}\left(\left\{\xi_{0}\right\} \times I\right)=$ $\eta_{\xi_{0}}\left(\left\{\xi_{0}\right\} \times I\right)=\{e\}$ and $I$ is compact there exists a neighborhood $Q_{\xi_{0}}$ of $\xi_{0}$ in $F$ so that $\sigma_{\xi_{0}}\left(Q_{\xi_{0}} \times I\right), \eta_{\xi_{0}}\left(Q_{\xi_{0}} \times I\right) \subset U$. Moreover, we have

$$
\begin{gathered}
\sigma_{\xi_{0}}(\cdot, 0)=\eta_{\xi_{0}}(\cdot, 0)=\text { const }_{e}, \\
\sigma_{\xi_{0}}(\xi, 1)=\eta_{\xi_{0}}(\xi, 1)=\exp \left(Z_{0}\right)^{-1} \cdot \exp (Z),
\end{gathered}
$$

where $\xi=(X, Y, Z) \in F$. Then, by means of a smooth contraction of $U$ we obtain a continuous map $S_{\xi_{0}}: Q_{\xi_{0}} \times I \times I \rightarrow U$ with smooth restrictions to each $Q_{\xi_{0}} \times I_{i} \times I$ so that

$$
\begin{gathered}
S_{\xi_{0}}(\cdot, \cdot, 0)=\sigma_{\xi_{0}}, \quad S_{\xi_{0}}(\cdot, \cdot, 1)=\eta_{\xi_{0}}, \\
S_{\xi_{0}}(\cdot, 0, \cdot)=\text { const }_{e}, \quad S_{\xi_{0}}(\xi, 1, s)=\exp \left(Z_{0}\right)^{-1} \cdot \exp (Z),
\end{gathered}
$$

for $\xi=(X, Y, Z) \in Q_{\xi_{0}}$.

Let $\bar{H}_{\xi_{0}}: Q_{\xi_{0}} \times I \times I \rightarrow \mathfrak{S}$ be the map defined by

$$
\bar{H}_{\xi_{0}}(\xi, t, s)=H_{\xi_{0}}(t, s) \cdot S_{\xi_{0}}(\xi, t, s) .
$$

Then it is easy to check that

$$
\begin{gathered}
\bar{H}_{\xi_{0}}(\cdot, \cdot, 0)=\sigma_{\mid \sigma_{\xi_{0} \times I}}, \quad \bar{H}_{\xi_{0}}(\cdot, \cdot, 1)=\eta_{\mid Q_{\xi_{0} \times I}}, \\
\bar{H}_{\xi_{0}}(\cdot, 0, \cdot)=\text { const }_{e}, \quad \bar{H}_{\xi_{0}}(\xi, 1, s)=\exp (Z),
\end{gathered}
$$

for $\xi=(X, Y, Z) \in Q_{\xi_{0}}$.

Since $F$ is compact there exist a finite number of points $\xi_{j}=\left(X_{j}, Y_{j}, Z_{j}\right) \in$ $F(j=1, \ldots, k)$ such that $F=Q_{\xi_{1}} \cup \cdots \cup Q_{\xi_{k}}$. Let $Q_{j}=Q_{\xi_{j}}$ and $\bar{H}_{j}=\bar{H}_{\xi_{j}}$. 
Lemma 3.1. For each $j$ there exists a unique continuous map $\widetilde{H}_{j}: \widetilde{M} \times Q_{j} \times I \times$ $I \rightarrow \widetilde{M}$ with smooth restrictions to each $\widetilde{M} \times Q_{j} \times I_{i} \times I$ such that

(i) $D \circ \widetilde{H}_{j}(\tilde{x}, \xi, t, s)=D(\tilde{x}) \cdot \bar{H}_{j}(\xi, t, s)$,

(ii) $\widetilde{H}_{j}(\tilde{x}, \xi, 0, s)=\tilde{x}$,

(iii) $(d / d t) \widetilde{H}_{j}(\tilde{x}, \xi, t, s) \in \tilde{\nu}$ for $t \neq 1 / 2$.

Proof. For $i=1,2$ and $j=1, \ldots, k$ we consider the smooth maps

$$
\begin{aligned}
J_{i, j}: \mathfrak{S} \times Q_{j} \times I_{i} \times I & \rightarrow G \\
(g, \xi, t, s) & \mapsto g \cdot \bar{H}_{j}(\xi, t, s) .
\end{aligned}
$$

We have the pull-back

$$
J_{i, j}^{*} \widetilde{M}=\left\{(g, \xi, t, s, \tilde{x}) \in \mathfrak{S} \times Q_{j} \times I_{i} \times I \times \widetilde{M} / D(\tilde{x})=g \cdot \bar{H}_{j}(\xi, t, s)\right\}
$$

of the fibre bundle $D: \widetilde{M} \rightarrow \mathfrak{S}$ with the projection $\bar{D}_{i, j}: J_{i, j}^{*} \widetilde{M} \rightarrow \mathfrak{G} \times Q_{j} \times I_{i} \times I$ given by $\bar{D}_{i, j}(g, \xi, t, s, \tilde{x})=(g, \xi, t, s)$ and the homomorphism of fibre bundles $\bar{J}_{i, j}: J_{i, j}^{*} \widetilde{M} \rightarrow \widetilde{M}$ given by $\bar{J}_{i, j}(g, \xi, t, s, \tilde{x})=\tilde{x}$.

It is easy to prove that for each $(g, \xi, t, s, \tilde{x}) \in J_{i, j}^{*} \widetilde{M}$ the homomorphism $\bar{J}_{i, j *}: T_{(g, \xi, t, s, \tilde{x})}\left(J_{i, j}^{*} \widetilde{M}\right) \rightarrow T_{\tilde{x}}(\widetilde{M})$ is surjective, then $\left(\bar{J}_{i, j *}\right)^{-1} \tilde{\nu} \subset T\left(J_{i, j}^{*} \widetilde{M}\right)$ is a vectorial subbundle, complementary of the vertical subbundle $\operatorname{Ker}\left(\bar{D}_{i, j *}\right) \subset$ $T\left(J_{i, j}^{*} \widetilde{M}\right)$. It follows that for the vector field $X_{i, j}=(0,0, \partial / \partial t, 0)$ on $\mathfrak{S} \times$ $Q_{j} \times I_{i} \times I$ there exists a unique vector field $\widetilde{X}_{i, j} \in \Gamma\left(\left(\bar{J}_{i, j *}\right)^{-1} \tilde{\nu}\right)$ on $J_{i, j}^{*} \widetilde{M}$ so that $\bar{D}_{i, j *} \circ \tilde{X}_{i, j}=X_{i, j} \circ \bar{D}_{i, j}$.

For $(\tilde{x}, \xi, t, s) \in \widetilde{M} \times Q_{j} \times I \times I$ we have that $(D(\tilde{x}), \xi, 0, s, \tilde{x}) \in J_{1, j}^{*} \widetilde{M}$ by (3.3), then we define $\widetilde{H}_{j}(\tilde{x}, \xi, t, s)=\bar{J}_{1, j} \circ\left(\widetilde{X}_{1, j}\right)_{t}(D(\tilde{x}), \xi, 0, s, \tilde{x})$ if $t \in I_{1}$. Let $\tilde{x}_{1}=\bar{J}_{1, j} \circ\left(\tilde{X}_{1, j}\right)_{1 / 2}(D(\tilde{x}), \xi, 0, s, \tilde{x}) \in \widetilde{M}$, then $\left(D(\tilde{x}), \xi, 1 / 2, s, \tilde{x}_{1}\right) \in$ $J_{2, j}^{*} \widetilde{M}$ and we define $\widetilde{H}_{j}(\tilde{x}, \xi, t, s)=J_{2, j} \circ\left(\tilde{X}_{2, j}\right)_{t-1 / 2}\left(D(\tilde{x}), \xi, 1 / 2, s, \tilde{x}_{1}\right)$ if $t \in I_{2}$. In this way $\widetilde{H}_{j}$ is continuous with smooth restrictions to each $\widetilde{M} \times Q_{j} \times I_{i} \times I$ and it is easy to check that (i)-(iii) is verified.

Now we prove the unicity of $\widetilde{H}_{j}$. Take $\tilde{x} \in \widetilde{M}, \xi \in Q_{j}$ and $s \in I$, and let $\gamma: I \rightarrow \widetilde{M}$ be a curve with smooth restrictions to each $I_{i}$ such that:

(i') $D \circ \gamma(t)=D(\tilde{x}) \cdot \bar{H}_{j}(\xi, t, s)$,

(ii') $\gamma(0)=\tilde{x}$

(iii') $(d / d t) \gamma(t) \in \tilde{\nu}$ for $t \neq 1 / 2$.

For $i=1,2$ we define the smooth maps $\bar{\gamma}_{i}: I_{i} \rightarrow J_{i, j}^{*} \widetilde{M}$ by setting $\bar{\gamma}_{i}(t)=$ $(D(\tilde{x}), \xi, t, s, \gamma(t))$ (it is well defined by $\left.\left(i^{\prime}\right)\right)$. We have $\bar{J}_{i, j} \circ \bar{\gamma}_{i}=\gamma_{\mid I_{i}}$, so $(d / d t) \bar{\gamma}_{i}(t) \in\left(\bar{J}_{i, j *}\right)^{-1} \tilde{\nu}$ for all $t \in I_{i}$ (by $\left.\left(\mathrm{iii}^{\prime}\right)\right)$. Moreover, $\bar{D}_{1, j} \circ \bar{\gamma}_{1}(t)=$ $\left(X_{1, j}\right)_{t}(D(\tilde{x}), \xi, 0, s)$ for $t \in I_{1}$ and $\bar{D}_{2, j} \circ \bar{\gamma}_{2}(t)=\left(X_{2, j}\right)_{t-1 / 2}(D(\tilde{x}), \xi, 1 / 2, s)$ for $t \in I_{2}$. Thus $\bar{\gamma}_{1}(t)=\left(\widetilde{X}_{1, j}\right)_{t}(D(\tilde{x}), \xi, 0, s, \tilde{x})$ for $t \in I_{1}$ and $\bar{\gamma}_{2}(t)=$ $\left(\widetilde{X}_{2, j}\right)_{t-1 / 2}(D(\tilde{x}), \xi, 1 / 2, \gamma(1 / 2))$ for $t \in I_{2}$, obtaining $\gamma=\tilde{H}(\tilde{x}, \xi, \cdot, s)$.

Lemma 3.2. For each $j$ and each $\xi=(X, Y, Z) \in Q_{j}$ we have

(i) $\widetilde{H}_{j}(\cdot, \xi, 1,0)=\tilde{\phi}_{Z}$,

(ii) $\tilde{H}_{j}(\cdot, \xi, 1,1)=\tilde{\phi}_{Y} \circ \tilde{\phi}_{X}$,

(iii) $\widetilde{H}_{j}(\tilde{x}, \xi, 1, s) \in D^{-1}(D(\tilde{x}) \cdot \exp (Z))$ for all $\tilde{x} \in \widetilde{M}$ and for all $s \in I$. 
Proof. Fix $\tilde{x} \in \widetilde{M}$ and consider the curves $\gamma_{0}, \gamma_{1}: I \rightarrow \widetilde{M}$ given by $\gamma_{0}(t)=$ $\widetilde{H}_{j}(\tilde{x}, \xi, t, 0)$ and $\gamma_{1}(t)=\widetilde{H}_{j}(\tilde{x}, \xi, t, 1)$. By (i) of Lemma 3.1 and (3.3) we have that $D \circ \gamma_{0}, D \circ \gamma_{1 \mid I_{1}}$ and $D \circ \gamma_{1 \mid I_{2}}$ are integral curves of the vector fields $Z, 2 X$ and $2 Y$ respectively. So, by (ii) and (iii) of Lemma 3.1 we have that $\gamma_{0}, \gamma_{1 \mid I_{1}}$ and $\gamma_{1 \mid I_{2}}$ are integral curves of $\widetilde{Z}^{\nu}, 2 \widetilde{X}^{\nu}$ and $2 \widetilde{Y}^{\nu}$ with initial points $\tilde{x}, \tilde{x}$ and $\gamma_{1}(1 / 2)$ respectively, from which (i) and (ii) follows.

On the other hand, by (i) of Lemma 3.1 and by (3.3) we obtain

$$
D \circ \widetilde{H}_{j}(\tilde{x}, \xi, 1, s)=D(\tilde{x}) \cdot \exp (Z) .
$$
that

By the unicity of $\widetilde{H}_{j}$ and by the $h$-equivariance of $D$ it can be easily proved

$$
\zeta \circ \widetilde{H}_{j}(\tilde{x}, \xi, t, s)=\widetilde{H}_{j}(\zeta(\tilde{x}), \xi, t, s)
$$

for all $\zeta \in \operatorname{Aut}(\pi)$. Thus there exists a smooth map $K_{j}: M \times Q_{j} \times I \rightarrow M$ such that the following diagram is commutative

$$
\begin{array}{rr}
\widetilde{M} \times Q_{j} \times I \stackrel{\widetilde{H}_{j}(\cdot, \cdot, 1, \cdot)}{\longrightarrow} \widetilde{M} \\
\pi \times \text { id } \times \text { id } \downarrow & \downarrow \pi \\
M \times Q_{j} \times I \stackrel{K_{j}}{\longrightarrow} & M
\end{array}
$$

Moreover, for all $\xi=(X, Y, Z) \in Q_{j}$, by Lemma 3.2 and by the commutativity of (1.7) and (3.5) we have that $K_{j}(\cdot, \xi, \cdot): M \times I \rightarrow M$ is an integrable homotopy of $\phi_{Z}$ to $\phi_{Y} \circ \phi_{Z}$ [E], therefore the corresponding homotopy operator on $A$ preserves the filtration, thus its bihomogeneous component of bidegree $(0,-1), k_{j, \xi}: A \rightarrow A$, verifies

$$
\left(\phi_{X}^{*} \circ \phi_{Y}^{*}-\phi_{Z}^{*}\right)_{0,0}=d_{0,1} \circ k_{j, \xi}+k_{j, \xi} \circ d_{0,1},
$$

and $k_{j, \xi}(\alpha)$ depends smoothly on $\xi \in Q_{j}$ for each $\alpha \in A$ fixed.

Take a partition of unity $\left\{f_{j}\right\}_{j=1, \ldots, k}$ subordinated to the open covering $\left\{Q_{j}\right\}_{j=1, \ldots, k}$ of $F$. Then the functions $f_{j}(X, \cdot, \cdot)$ form a partition of unity subordinated to the open covering of $F_{X}$ given by the subsets $Q_{j, X}=\{(Y, Z) \in$ $\left.\mathfrak{g}^{2} /(X, Y, Z) \in Q_{j}\right\} \subset F_{X}$.

For $\alpha \in A$ and $X \in \overline{B^{*}}$, by Proposition 2.3 we have

$$
\begin{gathered}
\phi_{X}^{*} \circ \rho(\alpha)=\int_{F_{X}} \phi_{X}^{*} \circ \phi_{Y}^{*} \alpha \cdot d \mu_{X}(Y, X), \\
\rho(\alpha)=\int_{W_{X,-X}} \phi_{Y}^{*} \alpha \cdot d \mu_{X}(Y, Z)=\int_{W_{-X, X}} \phi_{Y}^{*} \alpha \cdot d \mu_{-X}(Y, Z) \\
=\int_{F_{-X}} \phi_{Y}^{*} \alpha \cdot d \mu_{-X}(Y, Z)=\int_{F_{X}} \phi_{Z}^{*} \alpha \cdot d \mu_{X}(Y, Z),
\end{gathered}
$$

thus we get

$$
\left(\phi_{X}^{*} \circ \rho-\rho\right)(\alpha)=\int_{F_{X}}\left(\phi_{X}^{*} \circ \phi_{Y}^{*}-\phi_{Z}^{*}\right)(\alpha) \cdot d \mu_{X}(Y, Z)
$$


Therefore, if we define the bihomogeneous linear operator $\Psi_{X}: A \rightarrow A$ by setting

$$
\Psi_{X}(\alpha)=\sum_{j=1}^{k} \int_{Q_{j, X}} k_{j, \xi}(\alpha) \cdot f_{j}(\xi) \cdot d \mu_{X}(Y, Z),
$$

where $\xi=(X, Y, Z)$ for each $(Y, Z) \in Q_{j, X}$, by (3.6) and (3.7) we get

$$
\left(\phi_{X}^{*} \circ \rho-\rho\right)_{0,0}=d_{0,1} \circ \Psi_{X}+\Psi_{X} \circ d_{0,1} \text {. }
$$

Lemma 3.3. $\Psi_{X}(\alpha)$ depends continuously on $X \in \overline{B^{*}}$ for each $\alpha \in A$ fixed.

Proof. It is enough to prove that if $f: F \rightarrow \mathbb{R}$ is a continuous function then the function $\bar{f}: \overline{B^{*}} \rightarrow \mathbb{R}$ given by

$$
\bar{f}(X)=\int_{F_{X}} f(X, Y, Z) \cdot d \mu_{X}(Y, Z)
$$

is continuous. Take $X_{0} \in \overline{B^{*}}$ and let $\left(X_{n}\right)_{n \in \mathbb{N}}$ be a sequence in $\overline{B^{*}}$ converging to $X_{0}$. Let

$$
B_{0}=B \cap \bigcap_{m=0}^{\infty} L_{a_{m}}^{-1} B
$$

where $a_{m}=\exp \left(X_{m}\right)$, being $\mu\left(\mathfrak{S}-B_{0}\right)=0$. For $m=0,1,2, \ldots$ take the continuous function $f_{m}: B_{0} \rightarrow \mathbb{R}$ given by

$$
f_{m}(g)=f\left(X_{m}, \log (g), \log \left(a_{m} \cdot g\right)\right) .
$$

$\left(f_{n}\right)_{n \in \mathbb{N}}$ converges pointwise to $f_{0}$ and for each $n \in \mathbb{N}$ we have $\left|f_{n}(g)\right| \leq$ $\max \{|f(\xi)| / \xi \in F\}$ for all $g \in B_{0}$, therefore, by Lebesgue's Dominated Convergence Theorem and by Proposition 2.3 we obtain

$$
\lim _{n \rightarrow \infty} \bar{f}\left(X_{n}\right)=\lim _{n \rightarrow \infty} \int_{B_{0}} f_{n} \cdot d \mu=\int_{B_{0}} f_{0} \cdot d \mu=\bar{f}\left(X_{0}\right) .
$$

Lemma 3.4. For $\alpha \in A, X \in \mathfrak{g}$ and $t \in \mathbb{R}$ we have

$$
\phi_{t X}^{*} \alpha=\alpha+\int_{0}^{t} \phi_{s X}^{*} \circ \theta_{X} \alpha \cdot d s=\alpha+\theta_{X} \int_{0}^{t} \phi_{s X}^{*} \alpha \cdot d s .
$$

Proof. For each $x \in M$ take the smooth curve $\gamma_{x}: \mathbb{R} \rightarrow \Lambda T_{x}^{*} M$ given by

$$
\gamma_{x}(t)=\left(\phi_{t X}^{*} \alpha-\alpha-\int_{0}^{t} \phi_{s X}^{*} \alpha \cdot d s\right)(x) .
$$

We have $(d / d t) \gamma_{k}(t)=0$ for all $t \in \mathbb{R}$, so $\gamma_{x}(t)=\gamma_{x}(0)=0$.

Take $\alpha \in \operatorname{Ker}\left(d_{0,1}\right)$ determining $[\alpha] \in E_{1}$ (by (1.2)). If $[\alpha] \in \rho_{1}\left(E_{1}\right)$ we can suppose $\alpha=\rho_{0,0}(\beta)$ for some $\beta \in \operatorname{Ker}\left(d_{0,1}\right)$. Then, by (3.8) we have $\left(\phi_{X}^{*}\right)_{0,0} \alpha-\alpha=d_{0,1} \circ \Psi_{X}(\beta)$ for all $X \in \overline{B^{*}}$. Thus, by Lemma 3.3 and Lemma 3.4 it follows that

$$
\begin{aligned}
\left(\theta_{X}\right)_{0,0} \alpha & =\left(\theta_{X}\right)_{0,0} \int_{0}^{1}\left(\left(\phi_{s X}^{*}\right)_{0,0} \alpha-d_{0,1} \circ \Psi_{s X}(\beta)\right) \cdot d s \\
& =\left(\phi_{X}^{*}\right)_{0,0} \alpha-\alpha-d_{0,1} \circ\left(\theta_{X}\right)_{0,0} \int_{0}^{1} \Psi_{s X}(\beta) \cdot d s \\
& =d_{0,1}\left(\Psi_{X}(\beta)-\left(\theta_{X}\right)_{0,0} \int_{0}^{1} \Psi_{s X}(\beta) \cdot d s\right) .
\end{aligned}
$$


Therefore $\rho_{1}\left(E_{1}\right) \subset\left(E_{1}\right)_{\theta_{1}=0}$ and we can consider $\rho_{1}: E_{1} \rightarrow\left(E_{1}\right)_{\theta_{1}=0}$.

Now take $\alpha \in \operatorname{Ker}\left(d_{0,1}\right)$ such that $[\alpha] \in\left(E_{1}\right)_{\theta_{1}=0}$. Then, since $\left(\theta_{X}\right)_{0,0}$ depends linearly on $X \in \mathfrak{g}$ there exists a linear map $X \mapsto \beta_{X}$ of $\mathfrak{g}$ to $A$ so that $\left(\theta_{X}\right)_{0,0} \alpha=d_{0,1}\left(\beta_{X}\right)$ for all $X \in \mathfrak{g}$. Thus, by Lemma 3.4 we get

$$
\rho_{0,0}(\alpha)=\alpha+d_{0,1} \int_{\overline{B^{*}}} \int_{0}^{1}\left(\phi_{s X}^{*}\right)_{0,0} \beta_{X} \cdot d s \cdot \Delta^{*}(X),
$$

obtaining $\rho_{1}([\alpha])=[\alpha]$. Therefore, if $\imath:\left(E_{1}\right)_{\theta_{1}=0} \rightarrow E_{1}$ is the inclusion map we have $\rho_{1} \circ l=\mathrm{id}$. We also have $l \circ \rho_{1}-\mathrm{id}=d_{1} \circ \lambda_{1}+\lambda_{1} \circ d_{1}$ obtaining

$$
l_{*}=\rho_{1 *}^{-1}: H\left(\left(E_{1}\right)_{\theta_{1}=0}\right) \stackrel{\cong}{\longrightarrow} E_{2} \text {. }
$$

Since $\mathfrak{S}$ is compact $\theta_{\mathfrak{g}}$ is a semisimple representation ( $\S \S 4.4$ and 5.12 in Volume III of [GHV]), so by (1.6) and by results of Volume III of [GHV] (Theorem $\mathrm{V}$ of $\S 4.11$ and $\S 5.26$ ) it follows that

$$
H\left(\left(E_{1}\right)_{\theta_{1}=0}\right) \cong E_{2}^{0, \cdot} \otimes H^{\cdot}(\mathfrak{g}) .
$$

Therefore, from (3.10) and (3.11) we get

Theorem 3.5. Let $\mathfrak{g}$ be a compact semisimple Lie algebra. For Lie $\mathfrak{g}$-foliations on compact manifolds we have $E_{2}^{u, v} \cong E_{2}^{0, v} \otimes H^{u}(\mathfrak{g})$ for $u, v$ integers.

Remark. One of the main geometrical properties related to the spectral sequence associated to an orientable and oriented foliation $\mathscr{F}$ on a connected manifold $M$ is the minimality of the leaves for some Riemannian metric on $M:$ a smooth scalar product on $T \mathscr{F}$ is induced by a Riemannian metric on $M$ for which all the leaves are minimal submanifolds if and only if the corresponding volume form on the leaves is the restriction to the leaves of a p-form which defines an element in $E_{2}^{0, p}[\mathrm{R}, \mathrm{Su}$ and $\mathrm{Ha} 1]$. This property is verified whenever $\mathscr{F}$ is an orientable Lie $\mathfrak{g}$-foliation with $\mathfrak{g}$ compact or nilpotent and $M$ is compact [Ha 1 and $\mathrm{Ha} 2$ ]. In particular it is verified under the hypothesis of the above theorem when the foliation is orientable, and in this case we obtain that if $\chi$ is the characteristic form associated to any Riemannian metric on $M$ and any orientation of $\mathscr{F}$ [R] then the volume form along the leaves given by the restriction of $\rho(\chi)$ is induced by the orientation and the restriction to the leaves of a Riemannian metric on $M$ for which all the leaves of $\mathscr{F}$ are minimal submanifolds (because $\left.\left[\rho_{0,0}(\chi)\right] \in\left(E_{1}^{0, p}\right)_{\theta_{1}=0}=E_{2}^{0, p}\right)$.

Remark. The operators $\rho_{1}$ and $\lambda_{1}$ used to prove Theorem 3.5 are continuous, so they induce operators on $\mathscr{E}_{1}$ obtaining in a similar way (with a slight alteration of (3.9)) the analogous decomposition of $\mathscr{E}_{2}$ [A2]. Thus, by (1.4) we have $E_{2} \cong \mathscr{E}_{2}$ canonically, and by (1.3) we get

Corollary 3.6. With the same hypothesis, if the manifold is oriented we have $E_{2}^{u, v} \cong E_{2}^{q-u, v} \cong E_{2}^{u, p-v} \cong E_{2}^{q-u, p-v}$ for $u, v$ integers.

\section{RIEMANNIAN FOLIATIONS WITH COMPACT SEMISIMPLE STRUCTURAL LIE ALGEBRA}

Theorem 4.1. For Riemannian foliations on compact connected manifolds with compact semisimple structural Lie algebra we have $E_{2} \cong \mathscr{E}_{2}$ canonically.

Proof. The proof follows with similar arguments to those in $\S 8$ of [A2], they are shown here by the sake of completeness. 
Let $(M, \mathscr{F})$ be a foliated manifold verifying the hypothesis and let $\mathfrak{g}$ be its structural Lie algebra. Firstly assume that $\mathscr{F}$ is transversely parallelizable and let $\pi_{b}: M \rightarrow W$ be the basic fibre bundle [Mo]. The fibres of $\pi_{b}$ are the closures of the leaves of $\mathscr{F}$, and $\mathscr{F}$ induces on each fibre a Lie $\mathfrak{g}$-foliation.

We define the presheaves $\mathscr{P}_{i}$ and $\mathscr{Q}_{i}(i=1,2)$ by setting

$$
\mathscr{P}_{i}(U)=E_{i}\left(\mathscr{F}_{\mid \pi_{b}^{-1}(U)}\right) \quad \text { and } \quad \mathscr{Q}_{i}(U)=\mathscr{E}_{i}\left(\mathscr{F}_{\mid \pi_{b}^{-1}(U)}\right)
$$

for each open set $U$ in $W$, and with the canonical restrictions. Then, fixed a good open covering $\mathscr{U}$ of $W$ we have the Čech graded differential spaces $\left(\check{C}\left(\mathscr{U}, \mathscr{P}_{i}\right), \delta\right)$ and $\left(\check{C}\left(\mathscr{U}, \mathscr{Q}_{i}\right), \delta\right)$, and the differential operators $D=\delta+$ $(-1)^{k} d_{1}$ on $\check{C}^{k}\left(\mathscr{U}, \mathscr{P}_{1}\right)$ and $D^{\prime}=\delta+(-1)^{k} d_{1}$ on $\check{C}^{k}\left(\mathscr{U}, \mathscr{Q}_{1}\right)$, where $d_{1}$ is the differential operator on $\mathscr{E}_{1}$.

With a slight sharpening of the arguments of the Propositions 8.5 and 8.8 of [BT] we obtain that the restriction maps induce the isomorphisms $E_{2}(\mathscr{F}) \cong$ $H\left(\check{C}\left(\mathscr{U}, \mathscr{P}_{1}\right), D\right)$ and $\mathscr{E}_{2}(\mathscr{F}) \cong H\left(\check{C}\left(\mathscr{U}, \mathscr{Q}_{1}\right), D^{\prime}\right)$. Thus we obtain spectral sequences converging to $E_{2}(\mathscr{F})$ and $\mathscr{E}_{2}(\mathscr{F})$ with second terms $H\left(\check{C}\left(\mathscr{U}, \mathscr{P}_{2}\right), \delta\right)$ and $H\left(\check{C}\left(\mathscr{U}, \mathscr{Q}_{2}\right), \delta\right)$ respectively $(\S 14$ of $[\mathrm{BT}])$. Moreover, since $\mathscr{U}$ is a good open covering and the result is verified by Lie $\mathfrak{g}$-foliations on compact manifolds (second remark of Theorem 3.5$)$ we obtain $H\left(\check{C}\left(\mathscr{U}, \mathscr{P}_{2}\right), \delta\right) \cong H\left(\check{C}\left(\mathscr{U}, \mathscr{Q}_{2}\right), \delta\right)$ canonically, so $E_{2}(\mathscr{F}) \cong \mathscr{E}_{2}(\mathscr{F})$.

In the general case, by standard arguments we can suppose that $\mathscr{F}$ is transversely oriented. Then let $\widehat{\mathscr{F}}$ be the horizontal lifting of $\mathscr{F}$ to the principal $S \mathrm{O}(q)$-bundle of oriented orthonormal transverse frames with the transverse Levi-Civita connection [Mo]. The associated operation $(\mathfrak{o}(q), i, \theta, A(\widehat{M}), \hat{d})$ with the associated algebraic connection induces operations $\left(\mathfrak{o}(q), i_{1}, \theta_{1}\right.$, $\left.E_{1}(\widehat{\mathscr{F}}), \hat{d}_{1}\right)$ and $\left(\mathfrak{o}(q), \overline{i_{1}}, \overline{\theta_{1}}, \mathscr{E}_{1}(\widehat{\mathscr{F}}), \hat{d}_{1}\right)$ with the corresponding algebraic connections, being [A1 and A2]

$$
\begin{array}{ll}
E_{2}(\widehat{\mathscr{F}}) \cong H\left(E_{1}(\widehat{\mathscr{F}})_{\theta_{1}=0}\right), & E_{2}(\mathscr{F}) \cong H\left(E_{1}(\widehat{\mathscr{F}})_{\theta_{1}=0, i_{1}=0}\right), \\
\mathscr{E}_{2}(\widehat{\mathscr{F}}) \cong H\left(\mathscr{E}_{1}(\widehat{\mathscr{F}})_{\overline{\theta_{1}}=0}\right), & \mathscr{E}_{2}(\mathscr{F}) \cong H\left(\mathscr{E}_{1}(\widehat{\mathscr{F}})_{\overline{\theta_{1}}=0, \overline{i_{1}}=0}\right) .
\end{array}
$$

Thus, by results of Volume III of [GHV] (Corollary III of $\S 9.5$, Theorem III of $\S 5.18$ and Theorem I of $\S 5.12)$ there exist spectral sequences converging to $E_{2}(\widehat{\mathscr{F}})$ and $\mathscr{E}_{2}(\widehat{\mathscr{F}})$ with second terms $E_{2}(\widehat{\mathscr{F}}) \otimes H^{\bullet}(\mathfrak{o}(q))$ and $\mathscr{E}(\widehat{\mathscr{F}}) \otimes H^{\bullet}(\mathfrak{o}(q))$ respectively. Therefore, since $\widehat{\mathscr{F}}$ is transversely parallelizable the result follows by $\S 9.6$ of Volume III of [GHV] and by Theorem 11.1 of Chapter XI of [Mc].

Corollary 4.2. With the same hypothesis, if the manifold is oriented we have $E_{2}^{u, v} \cong E_{2}^{q-u, p-v}$ for $u$, $v$ integers.

\section{REFERENCES}

[A1] J. A. Alvarez Lopez, A finiteness theorem for the spectral sequence of a Riemannian foliation, Illinois J. Math. 33 (1989), 79-92.

[A2] __ Duality in the spectral sequence of Riemannian foliations, Amer. J. Math. 111 (1989), 905-926.

[B] N. Bourbaki, Integration, Hermann, Paris, 1969.

[BT] R. Bott and L. W. Tu, Differential forms in algebraic topology, Graduate Texts in Math., No. 82, Springer-Verlag, 1982. 
[E] A. El Kacimi-Alaoui, Sur la cohomologie feuilletée, Compositio Math. 49 (1983), 195-215.

[EH] A. El Kacimi-Alaoui and G. Hector, Décomposition de Hodge basique d'un feuilletage Riemannien, Ann. Inst. Fourier (Genoble) 36 (1986), 207-227.

[ESH] A. El Kacimi-Alaoui, V. Sergiescu, and G. Hector, La cohomologie basique d'un feuilletage Riemannien est de dimension finie, Math. Z. 188 (1985), 593-599.

[GHV] W. Greub, S. Halperin, and R. Vanstone, Connections, curvature and cohomology, Academic Press, 1973-1975.

[Ha1] A. Haefliger, Some remarks on foliations with minimal leaves, J. Differential Geometry 15 (1980), 269-284.

[Ha2] _ Pseudogroups of local isometries, Differential Geometry (L. A. Cordero, Ed.), Research Notes 131, Pitman, 1985, pp. 174-197.

[He] G. Hector, Cohomologies transversales des feuilletages Riemanniens, Feuilletages Riemanniens, Quantification Géométrique et Mécanique, Travaux en Cours, Hermann, Paris, 1988.

[Kl] W. Klingenberg, Riemannian geometry, De Gruyter Studies in Math., vol. 1, De Gruyter, 1982.

[Ko] S. Kobayashi, On conjugate and cut loci, Studies in Global Geometry and Analysis, Math. Assoc. of Amer., 1967, pp. 96-122.

[KT1] F. Kamber and Ph. Tondeur, Duality for Riemannian foliations, Proc. Sympos. Pure Math., vol. 40, part 1, Amer. Math. Soc., Providence, R.I., 1983, pp. 609-618.

[KT2] __ Foliations and metrics, Progress in Math., vol. 32, Differential Geometry, Birkhäuser, 1983, pp. 103-152.

[KT3] _ Duality theorems for foliations Astérisque 116 (1984), 108-116.

[Ma] X. Masa, Cohomology of Lie foliations, Differential Geometry, (L. A. Cordero, Ed.), Research Notes 131, Pitman, 1985, pp. 211-214.

[Mc] S. Mac Lane, Homology, Die Grundlehren der Math. Wissenschaften, No. 114, SpringerVerlag, 1963.

[Mo] P. Molino, Géométrie globale des feuilletages Riemanniens, Proc. Nederl. Akad. A1 85 (1982), 45-76.

[P] W. A. Poor, Differential geometric structures, McGraw-Hill, 1981.

[R] H. Rummler, Quelques notions simples en géométrie riemannienne et leurs applications aux feuilletages compacts, Comment. Math. Helv. 54 (1979), 224-239.

[Sa] K. S. Sarkaria, A finiteness theorem for foliated manifolds, J. Math. Soc. Japan 30 (1978), 687-696.

[Se1] V. Sergiescu, Cohomologie basique et dualité des feuilletages riemanniens, Ann. Inst. Fourier (Grenoble) 35 (1985), 137-158.

[Se2] _ _ Sur la suite spectrale d'un feuilletage Riemannien, Lille, 1986.

[Su] D. Sullivan, A homological characterization of foliations consisting of minimal surfaces, Comment. Math. Helv. 54 (1979), 218-223.

Departamento de Xeometria e Topoloxia, Facultad de Matemáticas, Universidad de Santiago de Compostela, 15705 Santiago de Compostela, Spain

Current address: Universidade de Santiago de Compostela, Colexio Universitario de Lugo, 27071 Lugo, Spain 